\author{
Knut Brockmann \\ Steffi Dreha-Kulaczewski \\ Peter Dechent \\ Carsten Bönnemann \\ Gunther Helms \\ Marten Kyllerman \\ Wolfgang Brück \\ Jens Frahm \\ Kathrin Huehne \\ Jutta Gärtner \\ Bernd Rautenstrauss
}

\section{Cerebral involvement in axonal Charcot-Marie-Tooth neuropathy caused by mitofusin 2 mutations}

Received: 28 May 2007

Received in revised form:

25 November 2007

Accepted: 12 December 2007

Published online: 21 April 2008

K. Brockmann (西) · S. Dreha-Kulaczewski · J. Gärtner

Dept. of Pediatrics and Pediatric Neurology Georg August University

Robert-Koch-Str. 40

37075 Göttingen, Germany

Tel.: +49-551/39-6210

Fax: +49-551/39-6252

E-Mail: kbrock@med.uni-goettingen.de

P. Dechent · G. Helms

MR Research in Neurology and Psychiatry

Georg August University

Göttingen, Germany

C. Bönnemann

Division of Neurology

The Children's Hospital of Philadelphia, and University of Pennsylvania

School of Medicine

Philadelphia, USA

M. Kyllerman

Dept. of Neuropediatrics

Queen Silvia Children's Hospital

Sahlgrenska University

Göteborg, Sweden

W. Brück

Dept. of Neuropathology

Georg August University

Göttingen, Germany
J. Frahm

Biomedizinische NMR Forschungs GmbH am Max-Planck-Institut für biophysika-

lische Chemie

Göttingen, Germany

K. Huehne • B. Rautenstrauss

Institute of Human Genetics

Friedrich Alexander University

of Erlangen-Nuremberg

Erlangen, Germany

B. Rautenstrauss

MGZ - Medical Genetics Center

Munich, Germany

Abstract Mutations in the mitofusin 2 (MFN2) gene are a major cause of primary axonal CharcotMarie-Tooth (CMT) neuropathy. This study aims at further characterization of cerebral white matter alterations observed in patients with MFN2 mutations. Molecular genetic, magnetic resonance imaging (MRI), magnetic resonance spectroscopy (MRS), and diffusion tensor imaging (DTI) investigations were performed in four unrelated patients aged 7 to 38 years with early onset axonal CMT neuropathy. Three distinct and so far undescribed MFN2 mutations were detected. Two patients had secondary macrocephaly and mild diffuse predominantly periventricular white matter alterations on MRI. In addition, one boy had symmetrical T2-hyperintensities in both thalami. Two patients had optic atrophy, one of them with normal MRI. In three patients proton MRS revealed elevated concentrations of total $\mathrm{N}$-acetyl compounds (neuronal marker), total creatine (found in all cells) and myo-inositol (astrocytic marker) in cerebral white and gray matter though with regional variation. These alterations were most pronounced in the two patients with abnormal MRI. DTI of these patients revealed mild reductions of fractional anisotropy and mild increase of mean diffusivity in white matter. The present findings indicate an enhanced cellular density in cerebral white matter of MFN2 neuropathy which is primarily due to a reactive gliosis without axonal damage and possibly accompanied by mild demyelination.

Key words hereditary motor and sensory neuropathy - CharcotMarie-Tooth disease - mitofusin . macrocephaly $\cdot$ magnetic resonance spectroscopy 


\section{Introduction}

Hereditary motor and sensory neuropathies (HMSN) or Charcot-Marie-Tooth syndromes are a group of clinically and genetically heterogeneous disorders of the peripheral nervous system. Charcot-Marie-Tooth (CMT) neuropathies have been divided into predominantly demyelinating (CMT1) and predominantly axonal (CMT2) forms based on electrophysiological and neuropathological features. Both conditions are associated with a large number of genetic defects. The demyelinating type 1 is most frequently caused by a $1.4 \mathrm{MB}$ tandem duplication comprising the PMP22 gene on chromosome 17p11.2-12 [28]. Mutations of the mitochondrial fusion protein mitofusin 2 gene, MFN2, on chromosome $1 \mathrm{p} 36.2$ have been recognized to be associated with CMT type 2A (CMT2A), an axonal form of Charcot-Marie-Tooth neuropathies [35]. MFN2 functions as a mitochondrial outer membrane GTPase. It regulates the mitochondrial network architecture by fusion of mitochondria and is necessary for structural integrity and mobility of mitochondria.

Subsequently, further families presenting clinical and electrophysiological features of CMT2A have been identified with mutations in the MFN2 gene $[8,11,19,20]$. Clinical symptoms in the majority of MFN2 defect patients reported until now are predominantly confined to the peripheral nervous system. The electrophysiological characteristics indicate a primary axonal neuropathy. In some families with CMT and MFN2 mutations pyramidal signs are present [33]. Furthermore, MFN2 mutations have been found in six families with optic atrophy and hereditary motor and sensory neuropathy (HMSN VI) [34].

A genotype/phenotype correlation in 29 patients with 22 distinct MFN2 mutations revealed two subgroups: a larger group with early onset and severe disease status, and a smaller group with later onset and milder clinical course [31]. In a recently reported series of 26 patients with CMT2A due to MFN2 mutations, subcortical white matter abnormalities were found by brain MRI in eight patients, most of them with late onset disease [9].

Here, we report involvement of the brain in four unrelated patients with early onset axonal CMT disease and so far undescribed MFN2 mutations. In three of these patients we performed combined magnetic resonance imaging (MRI), magnetic resonance spectroscopy (MRS), and diffusion tensor imaging (DTI) investigations to further characterize these white matter alterations.

\section{Subjects and methods}

\section{Subjects}

Four unrelated male patients aged 7 to 38 years were investigated. Clinical features are summarized in Table 1. All patients had onset of a slowly progressive motor and sensory polyneuropathy in childhood. Gait disturbance and foot deformation were present in all; hand function was mildly to moderately impaired in three patients. Exaggerated muscle stretch reflexes were found in patients 1 (until 15 years of age) and 3 , only the triceps surae reflex was absent. In patient 1, a saccadic interruption of horizontal eye movements was noted at 10 years of age, which prompted a cranial MRI investigation (see below). Optic atrophy was noted in patients 1 and 4 . Patient 1 reported visual impairment for the first time at 18 years of age, 15 years after onset of motor symptoms. At 17 years, with normal vision, stimulation with an alternating checkerboard pattern produced clearly identifiable VEP with P2-latencies at the upper limit of normal. One year later, neither pattern reversal nor flash stimulation produced an identifiable VEP. At this time, visual acuity was 0.5 (right eye) and 0.33 (left eye). Optic discs looked normal. Patient 4 had onset of motor symptoms at 3 years and complained increasing visual difficulties at 5 years of age. At 7.5 years, visual acuity was 0.1 , optic discs were pale and an afferent pupillar defect was present. VEP were not recorded, as the parents declined further investigations. Cranial nerves were otherwise normal in all patients.

Patients 1 and 2 had a secondary macrocephaly. In patient 1 , head circumference $(\mathrm{HC})$ grew from the $25^{\text {th }}$ percentile at 5 years to $2 \mathrm{~cm}$ above the $98^{\text {th }}$ percentile at 18 years of age (Fig. 1a). In patient 2 head growth followed the $75^{\text {th }}$ percentile until the age of 11 years, when a

Table 1 Clinical features

\begin{tabular}{lllll}
\hline Patient & 1 & 2 & 3 & 4 \\
\hline Gender & male & male & male & male \\
Present age (years) & 18 & 14 & 38 & 7 \\
Affected kinship & - & father & son & - \\
Onset of symptoms (years) & 3 & 7 & 7 & 3 \\
Loss of walking (years) & 10 & - & - & 6 \\
Hand function & impaired & impaired & normal & impaired \\
Optic atrophy (onset [years]) & $+(18)$ & - & - & $+(5)$ \\
Present head circumference & $>+2 S D$ & $>+2 S D$ & $+2 S D$ & normal \\
MFN2 amino acid change & p.R104W & IVS5-1G $>C$ & p.S378P & p.R104W \\
MRI & abnormal & abnormal & normal & normal \\
MRS/DTI & abnormal & abnormal & abnormal & n.t.
\end{tabular}

SD standard deviation; MRS magnetic resonance spectroscopy; DTI diffusion tensor imaging; n.t. not tested 
Fig. 1 Head growth of patient 1 (a) and patient 2 (b)

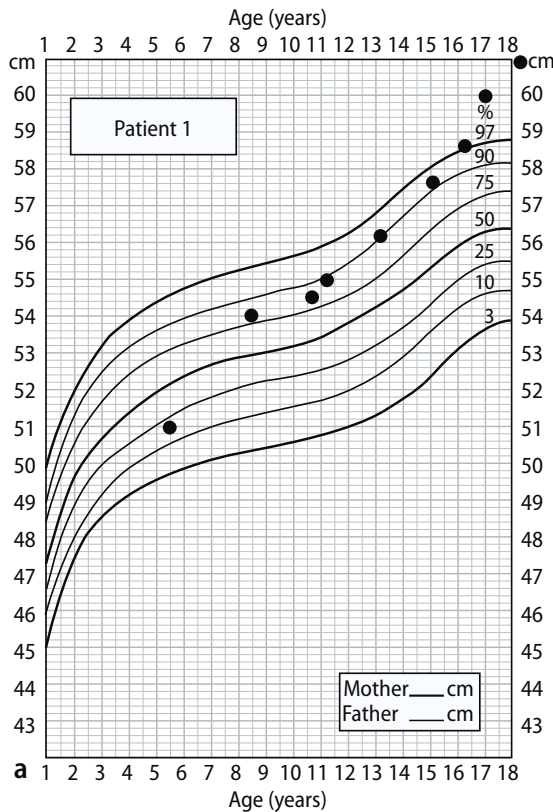

Age (years)

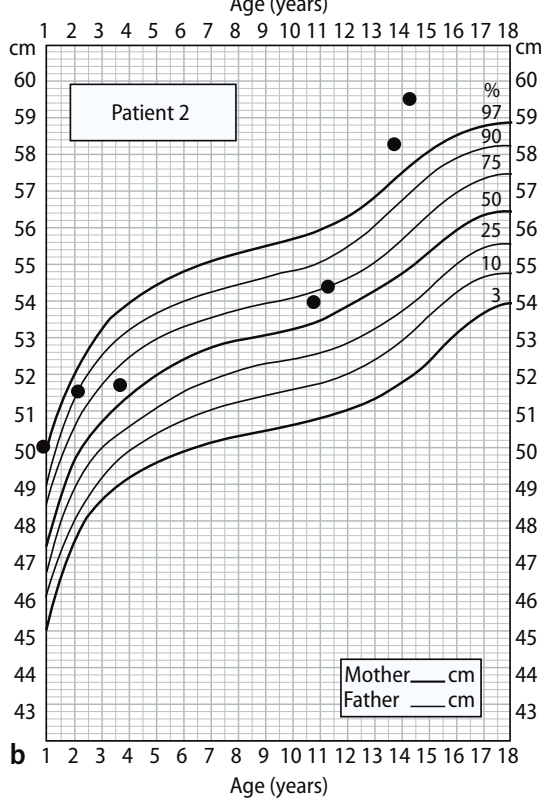

steep increase to a $\mathrm{HC}$ of $59.5 \mathrm{~cm}\left(1.5 \mathrm{~cm}>98^{\text {th }}\right.$ percentile $)$ at 14 years of age occurred (Fig.1b).

\section{Neurophysiological investigations}

Details of nerve conduction studies and evoked potentials are given in Table 2. In all patients, amplitudes of compound muscle action potentials and sensory nerve action potentials were markedly reduced, if recordable at all. Nerve conduction velocities were slightly reduced or normal. EEG showed slowing of background activity in patient 4 and was normal in the other patients.

\section{Histopathological investigations}

In patient 1 , a sural nerve biopsy at six years of age showed a loss of myelinated nerve fibers. There were numerous onion bulb formations present indicating repeated episodes of demyelination and remyelination. Numerous myelin sheaths were too thin in comparison to the axon diameter indicative of remyelination. There were no signs of acute axonal (Wallerian) degeneration or inflammation present (Fig.2). Electron microscopy confirmed the numerous onion bulbs. Non-myelinated axons did not show prominent signs of degeneration and there were no pathological inclusions found in Schwann cells.

In patient 4 , a sural nerve biopsy at six years of age revealed reduction of the larger myelinated fibers. No signs of ongoing atrophy in the remaining myelinated fibers were found. Signs of regeneration were present. In conclusion features of a primarily axonal neuropathy were detected.

\section{Methods}

\section{Genetic analysis}

Genomic DNA was extracted from peripheral blood samples by the salting-out method. DNA was diluted to a concentration of app. 50 $\mathrm{ng} / \mathrm{ml}$ and stored at $4{ }^{\circ} \mathrm{C}$. Primers flanking the exons of the mitofusin2 (MFN2) gene were created by using the Exon Locator program (http:// exon.swmed.edu/) of the BioSupplyNet. Oligonucleotide sequences and PCR conditions are available on request. The complete coding region of the exons and flanking intronic sequences were amplified. The purified PCR products were sequenced on both strands using the
BigDye Terminator Cycle Sequencing Kit (Applied Biosystems) on an ABI 3730 automated capillary sequencer (Applied Biosystems). The resulting sequences were compared to the reference sequences (MFN2, genomic contig: NT_021937,cDNA:NM_014874) given in the NCBI-database (http://www.ncbi.nlm.nih.gov) and sequence variations were documented. Sequence traces were evaluated using the DNAStar software package.

\section{Magnetic resonance investigations}

In patient $1, \mathrm{MRI}$ investigations were performed at 11 and 17 years of age at 1.5 T (Philips NT Gyrovision). Combined MRI and MR spectroscopy studies were carried out at $3 \mathrm{~T}$ (Siemens Magnetom Trio, Erlangen, Germany) using the standard eight channel phased-array headcoil at 17 years of age in patient 1 , at 14 years in patient 2 , and at 38 years in patient 3 . Written informed consent was obtained from patients and, in patients 1 and 2, from their parents. The study was approved by the Ethics Committee of the University Göttingen. In patient 4, MRI was carried out at $1.5 \mathrm{~T}$, additional MRS or DTI investigations were not performed.

Fully relaxed short-echo time proton MR spectra were acquired by means of a single-voxel stimulated echo acquisition mode (STEAM) localization sequence $(\mathrm{TR} / \mathrm{TE} / \mathrm{TM}=6000 / 20 / 10 \mathrm{~ms}, 64$ accumulations) as described previously [13,22].Volumes of interest (VOI) were selected from T1-weighted (3D MP-RAGE) and T2-weighted (3D TSE with variable flip angle) images and were located in affected frontal and parieto-occipital white matter (WM, $4.1 \mathrm{ml})$ and paramedian parietal gray matter (GM, $12.5 \mathrm{ml})$.

Spectral evaluation and quantification of absolute metabolite concentrations were accomplished with the use of LCModel [26]. The approach represents a user-independent fitting routine based on a library of calibrated model spectra of individual compounds and therefore avoids bias due to manual post-processing. Metabolite concentrations are expressed in $\mathrm{mM}$, i.e. mmol per liter VOI, without corrections for CSF contributions and residual T2 relaxation effects. Further details of MRS data acquisition and spectral evaluation have been described elsewhere [12]. A total of ten healthy subjects were examined and grouped according to their age to provide age-matched control values for each patient. Values deviating from the mean normal concentration by 2 SD or more were regarded as abnormal.

Major detectable metabolites include the neuroaxonal markers 
Table 2 Results of neurophysiological investigations in the four patients

A Motor nerve conduction studies

\begin{tabular}{lcclllllrr}
\hline Patient & Age & $\begin{array}{l}\text { Peroneal } \\
\mathrm{CV}(\mathrm{m} / \mathrm{s})\end{array}$ & $\begin{array}{l}\text { Peroneal } \\
\text { amplitude }(\mathrm{mV})\end{array}$ & $\begin{array}{l}\text { Tibial } \\
\mathrm{CV}(\mathrm{m} / \mathrm{s})\end{array}$ & $\begin{array}{l}\text { Tibial } \\
\text { amplitude }(\mathrm{mV})\end{array}$ & $\begin{array}{l}\text { Median } \\
\mathrm{CV}(\mathrm{m} / \mathrm{s})\end{array}$ & $\begin{array}{l}\text { Median } \\
\text { amplitude }(\mathrm{mV})\end{array}$ & $\begin{array}{l}\text { Ulnar } \\
\mathrm{CV}(\mathrm{m} / \mathrm{s})\end{array}$ & $\begin{array}{l}\text { Ulnar } \\
\text { amplitude }(\mathrm{mV})\end{array}$ \\
\hline 1 & 17 & 0 & 0 & 0 & 0 & 0 & 0 & 0 & 0 \\
2 & 14 & 44 & 0.6 & 35 & 0.2 & 59 & 14 & 63 & 12 \\
3 & 38 & 0 & 0 & 0 & 0 & 49 & 12 & 48 & 5 \\
4 & 7 & 0 & 0 & 0 & 0 & n.t. & n.t. & n.t. & n.t. \\
\hline
\end{tabular}

n.t. not tested

B Sensory nerve conduction studies

\begin{tabular}{lccccc}
\hline Patient & Age & $\begin{array}{l}\text { Sural } \\
\mathrm{CV}(\mathrm{m} / \mathrm{s})\end{array}$ & $\begin{array}{l}\text { Sural } \\
\text { amplitude }(\mu \mathrm{V})\end{array}$ & $\begin{array}{l}\text { Median } \\
\mathrm{CV}(\mathrm{m} / \mathrm{s})\end{array}$ & $\begin{array}{l}\text { Median } \\
\text { amplitude }(\mu \mathrm{V})\end{array}$ \\
\hline 1 & 17 & 0 & 0 & 0 & 0 \\
2 & 14 & 46 & 14 & 60 & 30 \\
3 & 38 & 0 & 0 & 36 & 2 \\
4 & 7 & 0 & 0 & n.t. & n.t. \\
\hline
\end{tabular}

n.t. not tested

\section{Evoked potentials}

\begin{tabular}{llllll}
\hline Patient & Age & BAEP & Median SSEP & Tibial SSEP & $\begin{array}{l}\text { VEP } \\
\text { P100 latencies, left/right eye }(\mathrm{ms})^{*}\end{array}$ \\
\hline 1 & 17 & $\mathrm{~N}$ & no SSEP identifiable & no SSEP identifiable & $\begin{array}{l}112 / 110 \\
\text { no VEP identifiable }\end{array}$ \\
2 & 18 & & & P1-latencies prolonged & $99 / 100$ \\
3 & 14 & N & N & no SSEP identifiable & $98 / 100$ \\
\hline
\end{tabular}

$C V$ conduction velocity; $N$ normal; $n$.t. not tested; * Pattern reversal stimulation, P100 latency: mean $101 \pm 3.5 \mathrm{~ms}$, normal $\leq 111.5 \mathrm{~ms}$

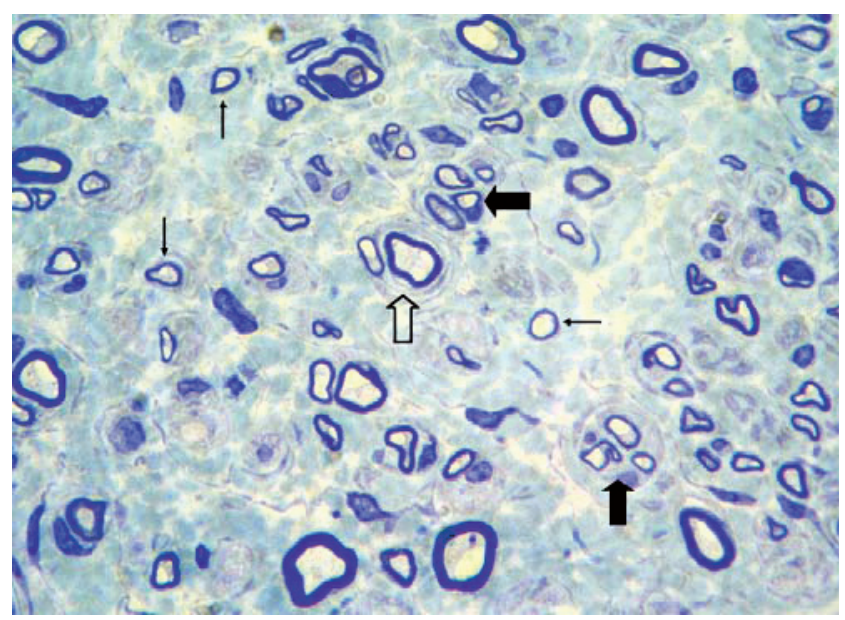

Fig. 2 Sural nerve biopsy of patient 1 at 4 years of age, toluidine blue stain, x100 magnification, shows a loss of myelinated nerve fibers with onion bulb formations, thin myelin sheaths in relation to axon diameter, and absence of any signs of acute axonal degeneration or inflammation. Thinly myelinated axons (small black arrows), onion bulb (big blank arrow), and regenerating clusters (big black arrows) are labeled
[4] N-acetylaspartate and N-acetylaspartylglutamate (tNAA), creatine and phosphocreatine ( $\mathrm{tCr}$ ) as ubiquitous compounds linked to energy metabolism [30], choline-containing compounds (Cho) involved in membrane turnover [15], the glial (astrocytic) marker myoinositol (Ins) [5], and lactate (Lac) as the intermediate product of nonoxidative glucose consumption.

Diffusion tensor imaging (DTI) was performed using a partial Fourier single-shot STEAM sequence [27]. In an axial orientation 38 consecutive sections of $2.2 \mathrm{~mm}$ thickness were acquired (TR $16.2 \mathrm{~s}$, TE $50 \mathrm{~ms}$, flip angle $10^{\circ}, 2.2 \times 2.2 \mathrm{~mm}^{2}$ in-plane resolution, $3-4$ averages), comprising a non-diffusion-weighted image and 24 images with diffusion-weighting in two sets of 12 directions with opposite sign (icosahedron scheme), at a b-value of $1000 \mathrm{~s} / \mathrm{mm}^{2}$. Maps of the mean diffusivity (MD) and the fractional anisotropy (FA) were calculated from the diffusion tensor and co-registered with the anatomical images using FSL 3.2 (FMRIB Software Library, Oxford). In a region-of-interest analysis MD- and FA-values were determined for the VOIs used for localized MRS of white matter as well as for the centrum semiovale. These values were compared to age-matched control values obtained from the ten healthy subjects. 


\section{Results}

\section{Mutation analysis}

In the mitofusin 2 gene of patient 1 and 4 , who are not related, we detected the heterozygous variation cytosine to thymine (c.310 C>T) in the second coding exon. The consequence is a change of arginine at position 104 to tryptophan (p.R104W). This variation was not detected in the patient's healthy parents and thus occurred de novo for patient 1 . The parents of patient 4 were not available for genetic analysis.

In patient 2 the mutation IVS5-1G $>C$ was identified heterozygous. This variation affects the canonic splice acceptor site. The same mutation was found in his mildly affected father, whereas his healthy mother carried the G nucleotide in both alleles required for splicing.

In patient 3 the heterozygous mutation thymine to cytosine (c.1132 $\mathrm{T}>\mathrm{C})$ in the ninth coding exon was found. The consequence is a change of the amino acid serine at position 378 to proline (p.S378P). This is also a de novo mutation, since the healthy parents carry the wildtype allele.

All three identified patient mutations were not found in 100 healthy controls. Furthermore, these mutations have not been described previously.

\section{Magnetic resonance imaging}

In patient $1, \mathrm{MRI}$ at 11 years revealed a mild diffuse hyperintensity of supratentorial white matter in T2- and FLAIR-weighted images. These signal alterations were diffuse but more prominent periventricularly in the parieto-occipital regions. A follow-up investigation at 17 years showed these hyperintensities unchanged (Fig.3). In patient 2 at 14 years of age, MRI showed mild signal alterations of predominantly periventricular parietooccipital white matter similar to the pattern in patient 1. In addition, symmetrical T2-hyperintensities were visible in lateral parts of both thalami (Fig.3e). MRI was normal in patient 3 at 38 years and in patient 4 at 7 years of age.

\section{Magnetic resonance spectroscopy}

MR spectra showed no gross abnormalities in any of the investigated patients (Fig.4). However, absolute quantification of metabolite concentrations (Table 3 ) revealed elevated concentrations of tNAA and tCr in all three patients though with regional variation. Of the two patients (1 and 2) with mild, predominantly parieto-occipital leukoencephalopathy on MRI, only patient 1 had moderate elevations of tNAA $(+20 \%), \mathrm{tCr}(+28 \%)$, and marked increase of Ins $(+67 \%)$ in the affected region as well as elevated Ins $(+30 \%)$ in frontal WM. In both pa-
Fig. 3 T2-weighted (a, b) and FLAIRweighted $(\mathbf{b}, \mathbf{d}) \mathrm{MRI}(1.5 \mathrm{~T})$ of patient 1 at $11(\mathbf{a}, \mathbf{b})$ and 17 years $(\mathbf{c}, \mathbf{d})$ of age shows non-progressive mild diffuse hyperintensity of supratentorial white matter predominantly in the periventricular regions. E T2-weighted axial MRI of patient 2 at 14 years of age showing stripes of hyperintensity in the lateral parts of both thalami
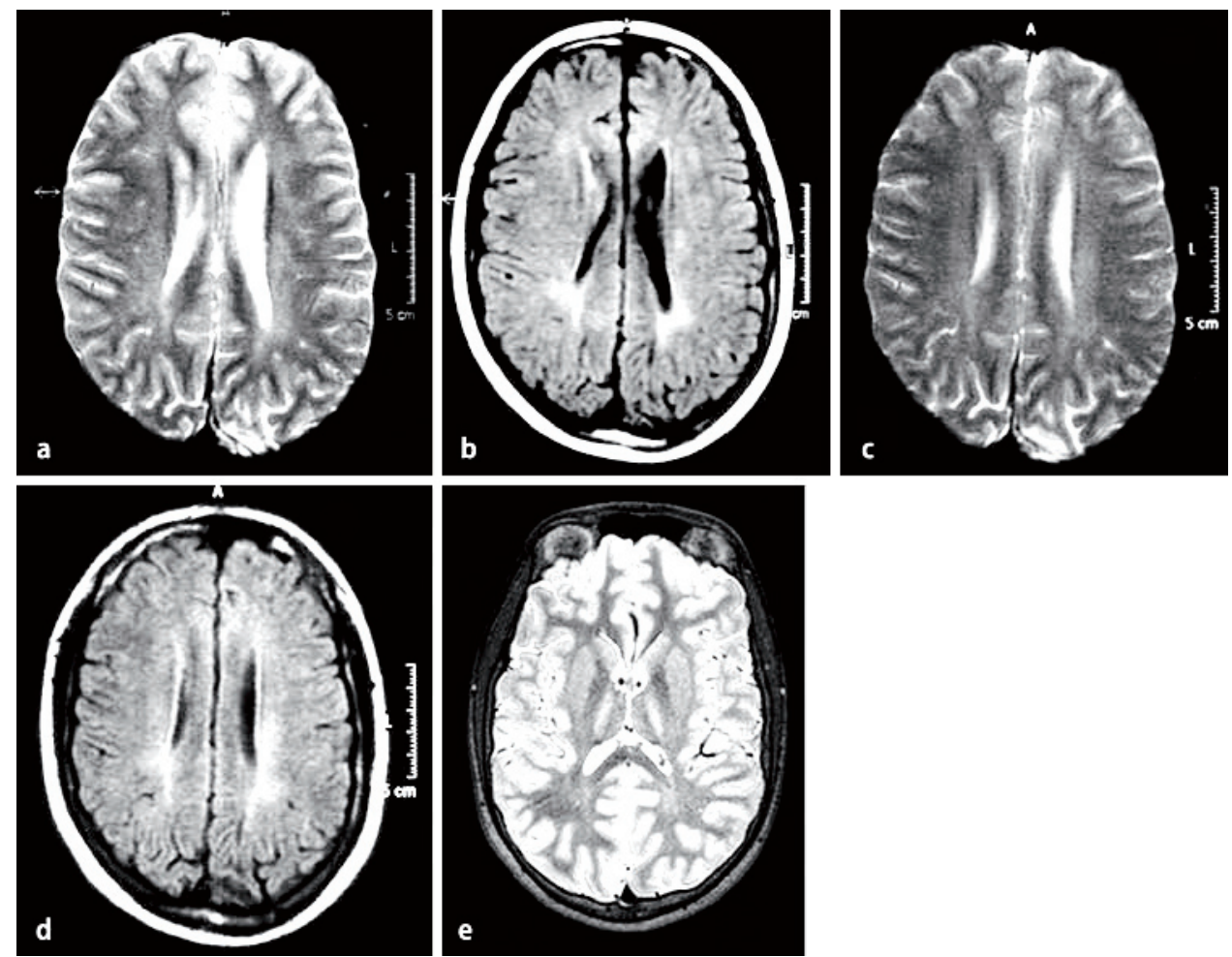


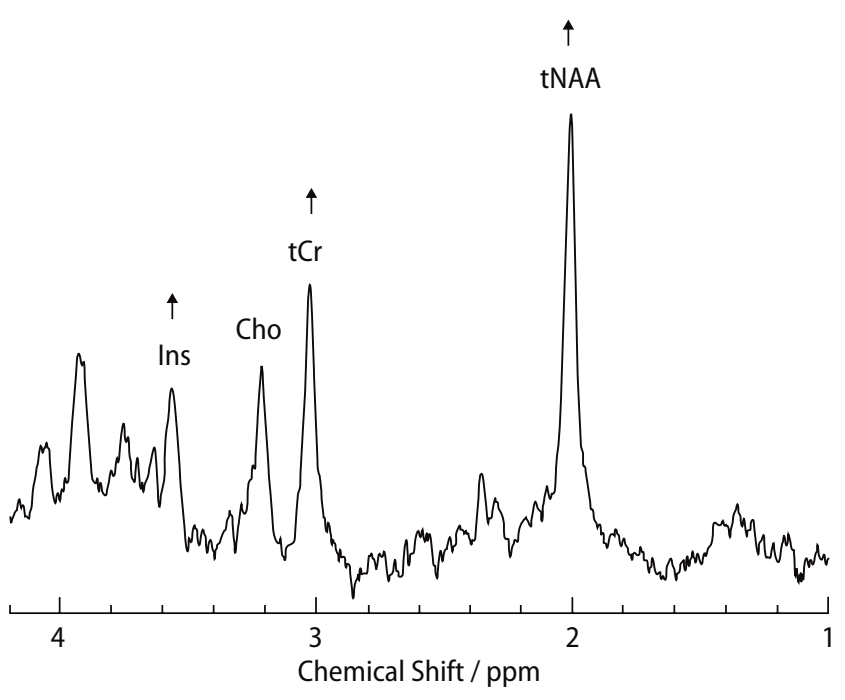

Fig. 4 Proton MRS (STEAM, TR/TE/TM $=6000 / 20 / 10 \mathrm{~ms}$ ) of right parieto-occipital white matter of patient 1 at 17 years of age

Table 3 Cerebral metabolite concentrations $(\mathrm{mM})$ in white and gray matter and age-matched controls (mean \pm SD)

\begin{tabular}{|c|c|c|c|c|c|}
\hline Patient & Region & tNAA & $\mathrm{tCr}$ & Cho & Ins \\
\hline 1 & WM LF & 6.2 & 4.6 & 1.7 & 3.5 \\
\hline 2 & WM LF & 6 & 3.9 & 1.7 & 2.9 \\
\hline \multicolumn{2}{|c|}{ Control ( $n=6,11-21$ yrs) } & $6.3 \pm 0.9$ & $4.2 \pm 0.7$ & $1.6 \pm 0.6$ & $2.7 \pm 0.6$ \\
\hline 1 & WM RPO & $9.1^{*}$ & $5.5^{*}$ & 1.6 & $4.5^{*}$ \\
\hline 2 & WM RPO & 7.5 & 4.3 & 1.5 & 2.9 \\
\hline \multicolumn{2}{|c|}{ Control ( $n=6,11-21$ yrs) } & $7.6 \pm 0.6$ & $4.3 \pm 0.6$ & $1.6 \pm 0.5$ & $2.7 \pm 0.6$ \\
\hline 1 & GM PMP & $8.3^{*}$ & $6.1^{*}$ & 1.2 & 4.2 \\
\hline 2 & GM PMP & 8.1 & $6.2^{*}$ & 1.3 & 3.7 \\
\hline \multicolumn{2}{|c|}{ Control $(n=6,11-21$ yrs $)$} & $6.6 \pm 0.8$ & $4.9 \pm 0.6$ & $1.0 \pm 0.3$ & $3.7 \pm 1.1$ \\
\hline 3 & WM LF & $7.1^{*}$ & $5.8^{*}$ & $1.9^{*}$ & 4.4 \\
\hline \multicolumn{2}{|c|}{ Control ( $\mathrm{n}=3,35-45$ yrs) } & $6.1 \pm 0.5$ & $3.7 \pm 0.5$ & $1.5 \pm 0.2$ & $2.9 \pm 0.8$ \\
\hline 3 & WM RPO & $8.0^{*}$ & 4.3 & 1.6 & $3.8^{*}$ \\
\hline \multicolumn{2}{|c|}{ Control ( $\mathrm{n}=3,35-45$ yrs) } & $7.3 \pm 0.1$ & $4.1 \pm 0.6$ & $1.5 \pm 0.3$ & $2.8 \pm 0.4$ \\
\hline 3 & GM PMP & 6.8 & 5.1 & 1.1 & 3.2 \\
\hline Control ( & $=3,35-45 \mathrm{yrs})$ & $7.0 \pm 0.7$ & $5.0 \pm 0.1$ & $1.0 \pm 0.1$ & $3.2 \pm 0.1$ \\
\hline
\end{tabular}

WM white matter; $L F$ left frontal; RPO right parieto-occipital; GM gray matter; $P M P$ paramedian parietal; * $\geq 2$ SD from control

tients moderate elevations of tNAA ( $+26 \%$ and $+23 \%)$ and $\mathrm{tCr}(+26 \%$ and $+24 \%)$ were found in parietal GM. In patient 3 with normal MRI, MRS of WM revealed mild increase of tNAA in frontal $(+16 \%)$ and parieto-occipital $(+10 \%)$ regions together with marked increase of $\mathrm{tCr}$ $(+57 \%)$ in the frontal area. This was accompanied by clear elevation of Ins in both frontal $(+52 \%)$ and parieto-occipital $(+36 \%)$ regions. A sole increase of Cho $(+27 \%)$ was detected in frontal WM of patient 3 , all other Cho levels were within the normal range. Remark- ably, concentrations of lactate were within the normal range in all regions in all patients.

\section{Diffusion tensor imaging}

DTI revealed only mild deviations from age-matched controls, as summarized in Table 4 . We found mildly decreased FA ( $>2 \mathrm{SD})$ in centrum semiovale of both patients 1 and 2 with abnormal T2-hyperintensity of periventricular white matter. Deviations of FA in frontal and parieto-occipital white matter of these two patients were inconsistent, with a tendency to reduction. Fig. 5 depicts the FA map of patient 1 in comparison to an age-matched control. MD was increased in frontal white matter of both patients and in centrum semiovale of one of them. In patient 3 , who had normal MRI, DTI revealed a mild elevation of FA in frontal white matter but otherwise no significant deviations from controls for FA and MD.

\section{Discussion}

Our study provides evidence for an involvement of cerebral white matter or optic nerves or both in four patients with CMT2 due to MFN2 mutations. Novel observations reported here include (i) the appearance of secondary macrocephaly in two patients ( 1 and 2 ) in their second decade, (ii) clear histopathological signs of demyelination in addition to features of axonal neuropathy in the sural nerve biopsy of one patient (1), (iii) involvement of thalami in one patient (2), (iv) metabolic and micro-

Table 4 Fractional anisotropy (FA) and mean diffusivity (MD) in WM of our patients and age-matched controls (mean \pm SD)

\begin{tabular}{lrll}
\hline \multicolumn{1}{l}{ Patient } & \multicolumn{1}{l}{ Region } & FA & MD \\
\hline 1 & WM LF & $0.23^{*}$ & $0.79^{*}$ \\
2 & WM LF & $0.28^{*}$ & $0.72^{*}$ \\
Control $(n=6,12-21$ yrs $)$ & $0.26 \pm 0.01$ & $0.69 \pm 0.01$ \\
1 & WM RPO & 0.3 & 0.76 \\
2 & WM RPO & 0.27 & 0.73 \\
Control $(n=6,12-21$ yrs $)$ & $0.33 \pm 0.04$ & $0.74 \pm 0.02$ \\
1 & WM R CS & $0.33^{*}$ & $0.72^{*}$ \\
2 & WM R CS & $0.32^{*}$ & 0.69 \\
Control ( $n=6,12-21$ yrs $)$ & $0.36 \pm 0.01$ & $0.67 \pm 0.02$ \\
3 & WM LF & $0.28^{*}$ & 0.7 \\
Control $(n=3,35-45$ yrs $)$ & $0.25 \pm 0.01$ & $0.69 \pm 0.01$ \\
3 & WM RPO & 0.29 & 0.7 \\
Control ( $n=3,35-45$ yrs $)$ & $0.3 \pm 0.03$ & $0.74 \pm 0.03$ \\
3 & WM R CS & 0.27 & 0.66 \\
Control ( $n=3,35-45$ yrs $)$ & $0.3 \pm 0.02$ & $0.66 \pm 0.02$ \\
\hline
\end{tabular}

$W M$ white matter; $L F$ left frontal; $R P O$ right parieto-occipital; $R C S$ right centrum semiovale; ${ }^{*} \geq 2$ SD from control 
Fig.5 Axial maps of the fractional anisotropy (thresholded at $F A=0.17$ ) overlaid on T1-weighted MRI of patient $1(\mathbf{a}, \mathbf{b})$ and a healthy, age-matched control (c, d)
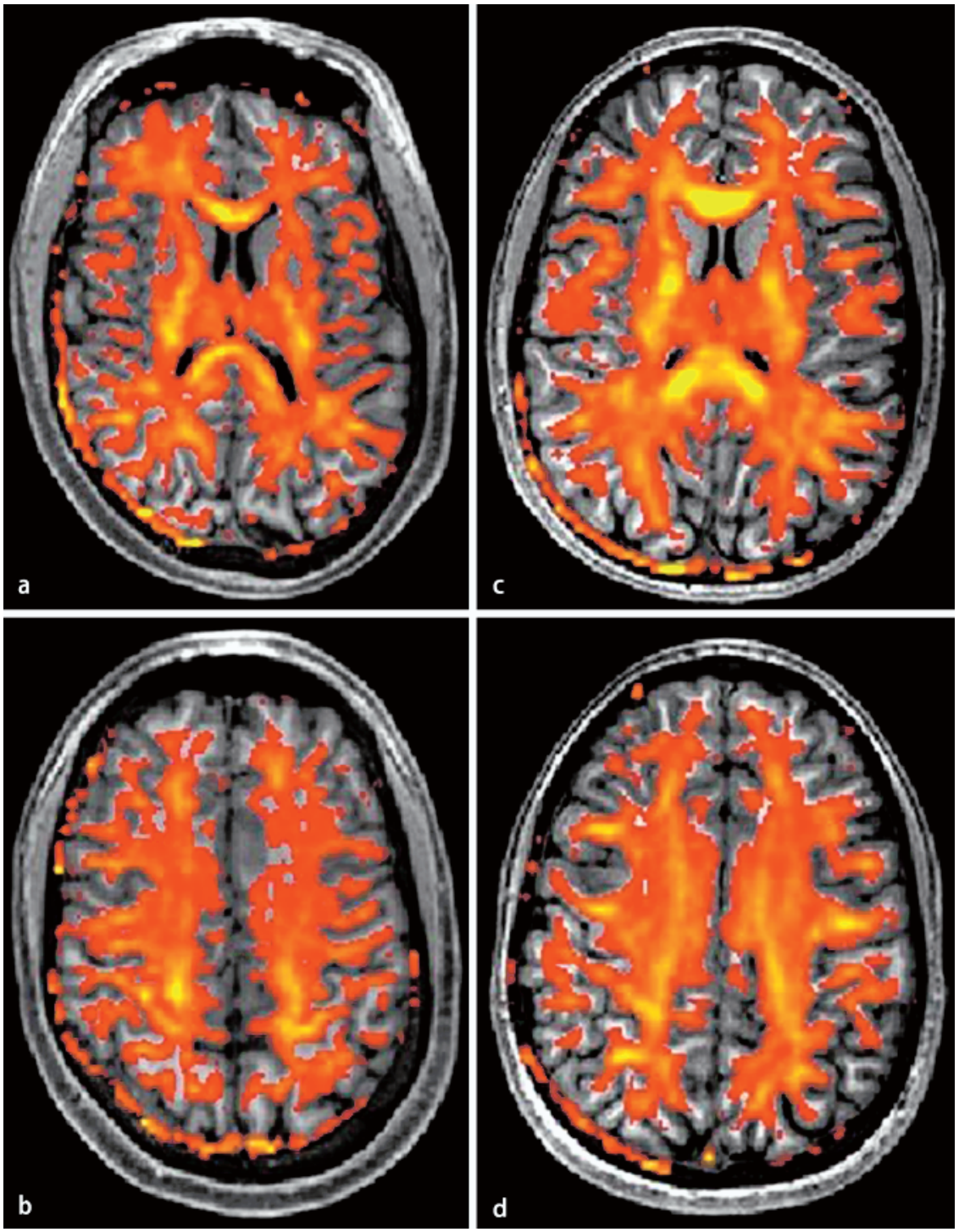

structural alterations detected by multiparametric MR investigations of cerebral white matter indicating enhanced neuroaxonal density, astrocytosis, and possibly mild demyelination in three patients (1-3), and (v) a splice site mutation in one patient (2) pointing to haploinsufficiency in MFN2 associated CMT.

The secondary macrocephaly in patients 1 and 2 (Fig. 1) constitutes a clinical feature of uncertain significance. This course of head growth is not typical for a familial macrocephaly, and, in patient 1 , it is not likely that this macrocephaly is due to the large HCs of both unaffected parents. At present, we can only presume that the relationship between MFN2 mutation and secondary macrocephaly in both boys is more likely to be cause and effect rather than mere coincidence. Remarkably, HC of patient 3 is also in the $98^{\text {th }}$ percentile range.

Reports on sural nerve biopsies in patients with MFN2 mutations are rare, and the histopathological findings are not uniform. The biopsies in the two patients presented here show features of axonal degeneration and additionally, in patient 1 , clear signs of repeated demyelination and remyelination with the formation of onion bulbs. These findings are compatible with the description of sural nerve pathology in a patient with late disease onset [9]. In this patient the authors show myelinated fibers being well preserved similar to our patient 1 . This is in contrast to the pathological features of a patient with early onset disease [9] and another patient described by Verhoeven et al. [31]. In these two cases a severe loss of myelinated fibers was observed with no evidence of regeneration or remyelination. Thus, the spectrum of sural nerve pathology in patients with MFN2 mutations may be broader than expected and may also include a remyelinating phenotype as described in the present patient beside the axonal degeneration reported previously. 
While MRI showed mild diffuse signal hyperintensities in T2- and FLAIR-weighted images in the two patients ( 1 and 2 ) with secondary macrocephaly and moderate to severe clinical course of peripheral neuropathy, MRI was normal in the older patient (3) with milder clinical features and in the 7-year-old boy (4) with signs of optic atrophy. In three patients, MRS revealed increases of tNAA, $\mathrm{tCr}$ and Ins with regional variation and to variable degree. DTI showed mild abnormalities in cerebral WM comprising decreased FA and elevated MD in the two patients with abnormal MRI.

We found no clear correlation between extent of T2signal changes on MRI and metabolic alterations detected by MRS. Clearly, however, MRS yielded no evidence for axonal loss in cerebral white matter that parallels the histological findings in the peripheral nerve. This is in line with the lack of clinical and neurophysiological features indicating marked CNS dysfunction. The only pyramidal tract signs that were present during the first years of the disease were exaggerated muscle stretch reflexes. Saccadic interruption of horizontal eye movements constituted the mild abnormality that prompted the MRI investigation of the brain in patient 1. Clinical examination, brainstem evoked potentials, and MRI provided no evidence for any brainstem or cerebellar abnormalities in this patient. One might speculate that disordered mitochondrial fusion in patients with MFN2 mutations could result in a change in NAA synthesis in neuronal mitochondria, but we have no evidence to support this hypothesis. The occurrence of secondary macrocephaly in patients 1 and 2 could be due to cerebral edema. However, this would result in more prominent T2-hyperintensity on brain MRI and reduced concentrations of major cerebral metabolites in MRS, as seen in laminin alpha2 chain deficiency [6]. On the other hand, macrocephaly may suggest an increase of volume of cerebral tissue. Astrocytic proliferation, as seen in Alexander disease, may result in secondary macrocephaly. Assuming changes in metabolite concentrations to reflect changes in cellular tissue composition rather than intracellular metabolism, the observation of increased tNAA (neuronal origin), $\mathrm{tCr}$ (all cells) and Ins (astrocytic origin) in our patients is best understood as the simultaneous presence of a pronounced astrocytic proliferation and enhanced neuroaxonal density.

MRI abnormalities and metabolic changes detected by MRS are complemented by only mild alterations of water diffusivity demonstrated by DTI. In general, white matter diffusion anisotropy reflects the integrity of both axonal membranes and myelin sheaths. Without any indication of axonal damage in proton MRS, the DTI findings hint to a mild demyelination which - together with the MRS alterations - seems to be accompanied by a more dense packing of axons (per image voxel) as well as a reactive gliosis. Thus, the present study suggests possible differences in the pathogenetic mechanisms of axonal CMT disease due to MFN2 mutations in peripheral and central nervous system.

Reports on involvement of cerebral white matter in CMT disease are rare. Preserved or increased muscle stretch reflexes and, in some cases, extensor plantar responses and increased muscle tone are features of HMSN type $\mathrm{V}$ according to Dyck $[14,18]$. MRI investigations of the brain have not been reported in these studies.

Disseminated lesions of subcortical white matter demonstrated by MRI of the brain have been described in single patients with hereditary neuropathy with liability to pressure palsies $[1,10]$. In CMT disease due to connexin 32 (Cx32, GJB1) mutations multifocal and in some cases transient and recurrent white matter lesions have been demonstrated by MRI of the brain $[17,21,23]$. In two male patients with X-linked CMT due to Cx32 mutations transient ataxia, dysarthria and weakness were associated with transient symmetrical white matter abnormalities on MRI. Additional proton MRS in one patient using metabolite ratios revealed a small decrease of tNAA/tCr ratio and elevated Cho/tCr ratios [24]. Further characterization using MR methods or histopathological studies of these white matter alterations are not available.

Neurological features of patients with MFN2 mutations reported previously are confined to the peripheral nervous system in most cases. A MFN2 mutation has been detected in a family with pyramidal signs [33] comprising extensor plantar responses, mild increase in tone, and preserved or increased reflexes but no spastic gait. Brain MRI was available from one member of this family and was normal [32]. MFN2 mutations were found in six families with HMSN VI, characterized by axonal neuropathy and optic atrophy [34]. Most of these patients had subacute onset of optic atrophy and subsequent slow recovery of visual acuity. No data regarding head circumferences are available. MRI or contrast-enhanced cranial CT scans were performed in at least one affected individual in five of the six families. These investigations were unremarkable in all but one patient, who had increased T2 signal in both cerebellar peduncles. Periventricular changes were not present [34].

More recently, a study of 26 patients from 15 families with CMT2A due to MFN2 mutations confirmed the delineation of an early onset group with severe clinical and electrophysiological features and a late onset group with milder clinical course [9]. In eight of these patients (seven in the late onset and only one in the early onset group), brain MRI disclosed abnormal, unspecific T2hyperintensities in subcortical and periventricular white matter. Data on head circumferences were not given.

A missense mutation p.R104W was found in our unrelated patients 1 and 4 . An exchange from arginine to tryptophan replaces an alkaline with a neutral amino acid residue. The arginine at position 104 is located within the GTPase domain of the MFN2 protein and is 
evolutionary highly conserved from the worm Chaenorhapditis elegans over the fruitfly Drosophila melanogaster up to homo sapiens. The severity of the clinical course of the neuropathy in both patients may be explained by a loss of function within the GTPase domain in MFN2. In both patients weakness started as early as 3 years of age and progressed rapidly, resulting in loss of walking at 10 years in patient 1 and at six years in patient 4. Both developed optic atrophy, patient 4 earlier than patient 1 . White matter changes on MRI were first detected in patient 1 at 11 years, and macrocephaly appeared only at 16 years. Follow-up investigations will reveal whether these features will appear in patient 4 , now aged seven years, later in the course. Histopathological investigation of sural nerve biopsies performed in both patients at the age of six years revealed features of axonal neuropathy in both boys and additional clear signs of demyelination in patient 1 .

In patient 3 with a milder clinical course we detected a missense mutation p.S378P. An exchange from serine to proline replaces a hydrophilic with a slightly polar amino acid residue. Proline is common in peptide bending or turns of the amino acid chain. The serine at position 378 of the MFN2 protein is conserved in mouse and rat and also in other GTPases like the transmembrane GTPase Fzo [16]. In D. melanogaster the serine at position 378 is replaced by asparagine, an uncharged polar amino acid, whereas the corresponding C. elegans protein also exhibits serine at the comparable position. Thus, the evolutionary conservation is not as strict as for the p.R104W variation in patients 1 and 4 . On the other hand the serine at position 378 is located in a conserved interdomain region. The amino acid position as well as the nature of the mutation in patient 3 point to less severe effects on the cellular level.

The mutation IVS5-1G $>C$ detected in patient 2 eliminates a canonic splice signal. Thus it can be predicted that exon 5 will be skipped and exon 6 is fused directly to exon 4. Under this assumption a frame shift results with a preliminary termination of translation at a tga codon in position 106. Hence the vast majority of the 757 amino acids of the wildtype protein is lost including the GTPase domain. This remaining protein fragment may be immediately degraded by proteolysis. Alternatively, one may assume a nonsense-mediated mRNA decay as a possible pathway. Thus, this mutation mimics a partial deletion or even a null allele of MFN2 reducing the effective dosage. In this context the associated phenotype corresponds to a haploinsufficiency due to a loss-offunction mutation.

Size and morphological arrangement of mitochondria are supposed to be the result of a dynamic balance between mitofusin-dependent mitochondrial fusion and dynamin-related protein dependent mitochondrial fission [29]. This balance varies with both cellular differentiation and human disease. MFN2 is a mitochondrial membrane protein that (i) mediates the fusion of mitochondria, and (ii) regulates mitochondrial membrane potential, fuel oxidation, and the OXPHOS system [25]. MFN2 is ubiquitously expressed [2] and mRNA transcripts have been detected in spinal cord and peripheral nerve [35]. It has been proposed that the metabolic accelerator and the mitochondrial fusion roles of MFN2 are distinct functions and that this may explain the sensitivity of the neuronal cell with its high energy demands to a partial MFN2 loss-of-function [25].MFN2deficient mice showed a reduced mitochondrial mobility [7], and a recent study demonstrated that disease-associated MFN2 mutant proteins induced impaired axonal mitochondrial transport in cultured dorsal root ganglion neurons [3]. Mobility and transport of mitochondria are crucial for the viability of long axons in both peripheral nerves and central tracts. However, the detailed mechanisms of MFN2 deficiency in CMT are poorly understood. Additional observations will elucidate whether secondary macrocephaly is a clinical hallmark of MFN2 neuropathy and further studies have to explain why MFN2 mutations result in primary axonal damage in the peripheral nerve versus astrocytic proliferation in cerebral white matter.

Acknowledgement We thank Dr. Göran Brandberg, Falun, Sweden, for referring patient 4. K.B. was supported by a grant from the German Bundesministerium für Bildung und Forschung (01GM0309). G.H. and P.D. were supported by a grant of the Volkswagen Stiftung. B.R. was supported by the Deutsche Forschungsgemeinschaft.

\section{References}

1. Amato AA, Barohn RJ (1996) Hereditary neuropathy with liability to pressure palsies: assocation with central nervous system demyelination. Muscle Nerve 19: 770-773
2. Bach D, Pich S, Soriano FX, Vega N, Baumgartner B, Oriola J, Daugaard JR, Lloberas J, Camps M, Zierath JR, Rabasa-Lhoret R, Wallberg-Henriksson $\mathrm{H}$, Laville M, Palacin M, Vidal H, Rivera F, Brand M, Zorzano A (2003) Mitofusin-2 determines mitochondrial network architecture and mitochondrial metabolism. A novel regulatory mechanism altered in obesity. J Biol Chem 278: 17190-17197
3. Baloh RH, Schmidt RE, Pestronk A, Milbrandt J (2007) Altered axonal mitochondrial transport in the pathogenesis of Charcot-Marie-Tooth disease from mitofusin 2 mutations. J Neurosci $27: 422-430$ 
4. Bjartmar C, Battistuta J, Terada N, Dupree E, Trapp BD (2002) N-acetylaspartate is an axon-specific marker of mature white matter in vivo: A biochemical and immunohistochemical study on the rat optic nerve. Ann Neurol 51:51-58

5. Brand A, Richter-Landsberg C, Leibfritz D (1993) Multinuclear NMR studies on the energy metabolism of glial and neuronal cells. Dev Neurosci 15:289-298

6. Brockmann K, Dechent P, Bönnemann C, Schreiber G, Frahm J, Hanefeld F (2007) Quantitative proton MRS of cerebral metabolites in Laminin $\alpha 2$ chain deficiency. Brain \& Development 29:357-364

7. Chen H, Detmer SA, Ewald AJ, Griffin EE, Fraser SE, Chan DC (2003) Mitofusins Mfn 1 and Mfn2 coordinately regulate mitochondrial fusion and are essential for embryonic development. J Cell Biol 160:189-200

8. Cho HJ, Sung DH, Kim BJ, Ki CS (2007) Mitochondrial GTPase mitofusin $2 \mathrm{mu}-$ tations in Korean patients with CharcotMarie-Tooth neuropathy type 2 . Clin Genet 71:267-272

9. Chung KW, Kim SB, Park KD, Choi KG, Lee JH, Eun HW, Suh JS, Hwang JH, Kim WK, Seo BC, Kim SH, Son IH, Kim SM, Sunwoo IN, Choi BO (2006) Early onset severe and late-onset mild CharcotMarie-Tooth disease with mitofusin 2 (MFN2) mutations. Brain 129: 2103-2118

10. Dackovic J, Rakocevic-Stojanovic V, Pavlovic S, Zamurovic N, Dragasevic N, Romac S, Apostolski S (2001) Hereditary neuropathy with liability to pressure palsies associated with central nervous system myelin lesions. Eur J Neurol 8:689-692

11. Engelfried $K$, Vorgerd $M$, Hagedorn $M$, Haas G, Gilles J, Epplen JT, Meins M (2006) Charcot-Marie-Tooth neuropathy type $2 \mathrm{~A}$ : novel mutations in the mitofusin 2 gene (MFN2). BMC Med Genet 7:53

12. Frahm J, Hanefeld F (1996) Localized proton magnetic resonance spectroscopy of cerebral metabolites. Neuropediatrics 27:64-69

13. Frahm J, Michaelis T, Merboldt KD, Bruhn H, Gyngell ML, Hänicke W (1990) Improvements in localized proton NMR spectroscopy of human brain. Water suppression, short echo times, and $1 \mathrm{~mL}$ resolution. J Magn Reson 90:464-473

14. Frith JA, McLeod JG, Nicholson GA, Yang F (1994) Peroneal muscular atrophy with pyramidal tract features (hereditary motor and sensory neuropathy type V): a clinical, neurophysiological, and pathological study of a large kindred. J Neurol Neurosurg Psychiatry 57:1343-1346
15. Griffin JL, Mann CJ, Scott J, Shoulders CC, Nicholson JK (2001) Choline containing metabolites during cell transfection: an insight into magnetic resonance spectroscopy detectable changes. FEBS Lett 509:263-266

16. Hales KG, Fuller MT (1997) Developmentally regulated mitochondrial fusion mediated by a conserved, novel, predicted GTPase. Cell 90:121-129

17. Hanemann CO, Bergmann C, Senderek J, Zerres K, Sperfeld AD (2003) Transient, recurrent, white matter lesions in X-linked Charcot-Marie-Tooth disease with novel connexin 32 mutation. Arch Neurol 60:605-609

18. Harding AE, Thomas PK (1984) Peroneal muscular atrophy with pyramidal features. J Neurol Neurosurg Psychiatry 47:168-172

19. Kijima K, Numakura C, Izumino $H$, Umetsu K, Nezu A, Shiiki T, Ogawa M, Ishizaki Y, Kitamura T, Shozawa Y, Hayasaka K (2005) Mitochondrial GTPase mitofusin 2 mutation in CharcotMarie-Tooth neuropathy type $2 \mathrm{~A}$. Hum Genet 116:23-27

20. Lawson VH, Graham BV, Flanigan KM (2005) Clinical and electrophysiologic features of CMT2A with mutations in the mitofusin 2 gene. Neurology 65 : 197-204

21. Lee MJ, Nelson I, Houlden H, Sweeney MG, Hilton-Jones D, Blake J, Wood NW, Reilly MM (2002) Six novel connexin32 (GJB1) mutations in X-linked CharcotMarie-Tooth disease. J Neurol Neurosurg Psychiatry 73:304-306

22. Natt $\mathrm{O}$, Bezkorovaynyy $\mathrm{V}$, Michaelis $\mathrm{T}$, Frahm J (2005) Use of phased array coils for a determination of absolute metabolite concentrations. Magn Reson Med 53:3-8

23. Panas M, Karadimas C, Avramopoulos D, Vassilopoulos D (1998) Central nervous system involvement in four patients with Charcot-Marie-Tooth disease with connexin 32 extracellular mutations. J Neurol Neurosurg Psychiatry 65:947-948

24. Paulson HL, Garbern JY, Hoban TF, Krajewski KM, Lewis RA, Fischbeck KH, Grossman RI, Lenkinski R, Kamholz JA, Shy ME (2002) Transient central nervous system white matter abnormality in X-linked Charcot-Marie-Tooth disease. Ann Neurol 52:429-434

25. Pich S, Bach D, Briones P, Liesa $M$, Camps M, Testar X, Palacin M, Zorzano A (2005) The Charcot-Marie-Tooth type 2A gene product, Mfn2, up-regulates fuel oxidation through expression of OXPHOS system. Hum Mol Genet 14: 1405-1415

26. Provencher SW (1993) Estimation of metabolite concentration from localized in vivo proton NMR spectra. Magn Reson Med 30:672-679
27. Rieseberg S, Merboldt KD, Kuntzel M, Frahm J (2005) Diffusion tensor imaging using partial Fourier STEAM MRI with projection onto convex subsets reconstruction. Magn Reson Med 54: 486-490

28. Saifi GM, Szigeti K, Snipes GJ, Garcia CA, Lupski JR (2003) Molecular mechanisms, diagnosis, and rational approaches to management of and therapy for Charcot-Marie-Tooth disease and related peripheral neuropathies. J Investig Med 51:261-283

29. Santel A, Fuller MT (2001) Control of mitochondrial morphology by a human mitofusin. J Cell Sci 114:867-864

30. Urenjak J, Williams SR, Gadian DG, Noble M (1993) Proton nuclear magnetic resonance spectroscopy unambiguously identifies different neural cell types. J Neurosci 13:981-989

31. Verhoeven K, Claeys KG, Zuchner S, Schroder JM, Weis J, Ceuterick C, Jordanova A, Nelis E, De Vriendt E, Van Hul M, Seeman P, Mazanec R, Saifi GM, Szigeti K, Mancias P, Butler IJ, Kochanski A, Ryniewicz B, De Bleecker J, Van den Bergh P, Verellen C, Van Coster R, Goemans N, Auer-Grumbach M, Robberecht W, Milic Rasic V, Nevo Y, Tournev I, Guergueltcheva V, Roelens F, Vieregge P, Vinci P, Moreno MT, Christen HJ, Shy ME, Lupski JR, Vance JM, De Jonghe P, Timmerman V (2006) MFN2 mutation distribution and genotype/phenotype correlation in Charcot-Marie-Tooth type 2. Brain 129:2093-2102

32. Vucic S, Kennerson M, Zhu D, Miedema E, Kok C, Nicholson GA (2003) CMT with pyramidal features. CharcotMarie-Tooth. Neurology 60:696-699

33. Zhu D, Kennerson ML, Walizada G, Zuchner S, Vance JM, Nicholson GA (2005) Charcot-Marie-Tooth with pyramidal signs is genetically heterogeneous: families with and without MFN2 mutations. Neurology 65:496-497

34. Zuchner S, De Jonghe P, Jordanova A, Claeys KG, Guergueltcheva V, Cherninkova S, Hamilton SR, Van Stavern G, Krajewski KM, Stajich J, Tournev I, Verhoeven K, Langerhorst CT, de Visser M, Baas F, Bird T, Timmerman V, Shy M, Vance JM (2006) Axonal neuropathy with optic atrophy is caused by mutations in mitofusin 2. Ann Neurol 59: 276-281

35. Zuchner S, Mersiyanova IV, Muglia M, Bissar-Tadmouri N, Rochelle J, Dadali EL, Zappia M, Nelis E, Patitucci A, Senderek J, Parman Y, Evgrafov O, Jonghe PD, Takahashi Y, Tsuji S, PericakVance MA, Quattrone A, Battaloglu E, Polyakov AV, Timmerman V, Schroder JM, Vance JM (2004) Mutations in the mitochondrial GTPase mitofusin 2 cause Charcot-Marie-Tooth neuropathy type 2A. Nat Genet 36:449-451 